\title{
Aportes al conocimiento de la riqueza florística para la gestión ambiental de la Sierra de Najasa, Camagüey, Cuba
}

\author{
Adelaida Barreto Valdés ${ }^{1 / 2}$ \\ Everardo Pérez Carreras 3/2 \\ Grisel Reyes Artiles ${ }^{4 / 2}$ \\ Néstor Enríquez Salgueiro ${ }^{5 / 2}$ \\ Josefa Primelles Fariñas ${ }^{6 / 2}$ \\ Erick Sedeño Bueno ${ }^{7 / 2}$
}

\begin{abstract}
RESUMEN
Se relacionan los aspectos más interesantes del recurso florístico de la elevación conocida como Sierra de Najasa en el municipio de Najasa, provincia Camagüey, Cuba. La riqueza total es de 132 géneros y 167 especies pertenecientes a 64 familias botánicas, lo que representa el 2.5\% de las plantas vasculares cubanas en el área estudiada. El mapa de vegetación confeccionado muestra las formaciones vegetales y las estaciones de muestreo. El número más alto de especies se encuentra en las estaciones 6,8 y 11 caracterizadas por dos variantes del bosque semideciduo. Los endémicos cubanos colectados representan el $8.6 \%$ respecto al total de especies muestreadas en el área y la estación 7 posee la mayor riqueza de endemismos. La relación florística más estrecha es con la flora neotropical (32.8\%), aunque el $22.5 \%$ de las especies son afines a la caribeana y el $12.2 \%$ a la antillana. El $76.3 \%$ de las especies tienen potencialidades como recurso natural aprovechable por el hombre con propósitos diferentes.
\end{abstract}

Palabras Claves: Plantas vasculares, Sierra de Najasa, Cuba

\begin{abstract}
The more interesting aspects of floristic resources of the Sierra de Najasa, Najasa municipality, Camagüey, Cuba are expressed. The total richness is 132 genera and 167 species in 64 families; this represents $2.5 \%$ of Cuban vascular plants in the area. The vegetation map shows the plant communities and the sampling sites. The highest number of species is found in the sites 6,8 and 11 , which are characterized by two kinds of semideciduous forest. Cuban endemics, that were colected, represents the $8.6 \%$ of the area flora and the site 7 showed the higher number of endemics. The principal floristic relationship is with the Neotropical flora (32.8\%), although $22.5 \%$ of species are common to the Caribbean flora and $12.2 \%$ to the Antillean flora. $76.3 \%$ of the species have potentialities as a natural resource that could be used by man for different purposes.
\end{abstract}

Keywords: Vascular plants, Sierra de Najasa, Cuba

\footnotetext{
${ }^{1}$ Investigador Titular del CIMAC, Ministerio de Ciencia, Tecnología y Medio Ambiente (CITMA)

${ }^{2}$ Centro Investigaciones del Medio Ambiente, CITMA -Cisneros 105, Camagüey CP 70100, Cuba.

${ }^{3}$ Investigador Auxiliar del CIMAC-CITMA

${ }^{4}$ Ingeniera en Sistemas Automatizados de Dirección, Especialista del CIMAC-CITMA

${ }^{5}$ Técnico Medio en Agronomía del CIMAC-CITMA

${ }^{6}$ Investigador Auxiliar del CIMAC-CITMA

${ }^{7}$ Aspirante a Investigador del CIMAC-CITMA
} 


\section{INTRODUCCIÓN}

La Sierra de Najasa se localiza en el municipio de Najasa al sur de la provincia políticoadministrativa de Camagüey, con una altura máxima de 301 m.s.n.m., y una superficie de 323 ha.

Borhidi \& Muñiz (1986) incluyeron esta Sierra en la subprovincia florística Cuba Central, sector Cuba Centro-Oriental, distrito Guaimarense, caracterizada por un clima tropical estacional, seco en el invierno y algo más húmedo hacia el sur de Camagüey. Estos autores señalaron las características cársicas de las alturas que conforman la Sierra de Najasa, así como la ocurrencia de suelo húmico-carbonatado.

Pérez et al. (1994) estudiaron la vegetación boscosa de esta localidad, conjuntamente con la de elevaciones vecinas, y describieron las formaciones vegetales «complejo de vegetación de mogote» con sus componentes vegetación de farallón y bosque semideciduo mesófilo, y «vegetación secundaria», donde las sabanas antrópicas prevalecen. Los autores relacionaron la flora, pero no particularizaron la presencia o ausencia de especies en una u otra elevación.

Este trabajo proveerá un conocimiento más profundo de los componentes de la flora de la altura conocida como Sierra de Najasa dentro del complejo orográfico de su nombre como parte de un proyecto de gestión ambiental que se ejecuta para el municipio Najasa, en función de lograr una política de desarrollo sustentable para la región.

\section{MATERIALES Y MÉTODOS}

Se seleccionaron 12 estaciones de la Sierra (Tabla 1) tomando en consideración la representatividad y el estado de conservación de las diferentes formaciones vegetales que la caracterizan, como criterios válidos para escoger sitios de acuerdo a Sobrevila y Bath (1992).

Las colectas se realizaron estableciendo

Tabla 1 - Riqueza de especies por estaciones, su representatividad y endemismo. PC=endemismo pancubano, COc$\mathrm{CC}=$ endemismo de Cuba Occidental-Cuba Central, $\mathrm{CC}=$ endemismo de Cuba Central, CC-COr=endemismo de Cuba Central-Cuba Oriental.

\begin{tabular}{|c|c|c|c|c|c|c|c|}
\hline \multirow[t]{2}{*}{ Estación } & \multirow[t]{2}{*}{ Denominación } & \multirow{2}{*}{$\begin{array}{l}\text { No. Especies/ } \\
\text { estación }\end{array}$} & \multirow{2}{*}{$\begin{array}{c}\text { \% representatividad } \\
\text { respecto a flora } \\
\text { del área }\end{array}$} & \multicolumn{4}{|c|}{ Endemismo/Estación } \\
\hline & & & & PC & COc-CC & $\begin{array}{l}\mathbf{C} \\
\mathbf{C}\end{array}$ & CC-COr \\
\hline 1 & Cima del Farallón Colorado & 17 & 10.2 & 6 & & & \\
\hline 2 & El Mirador (yayal) & 1 & 0.6 & & & & \\
\hline 3 & Callejones del Infirno & 9 & 5.4 & 1 & & & \\
\hline 4 & $\begin{array}{l}\text { Cerca de los Callejones del } \\
\text { Infierno }\end{array}$ & 18 & 10.7 & 2 & & 1 & \\
\hline 5 & $\begin{array}{l}\text { e/El Mirador y el Cocal } \\
\text { (cima loma) }\end{array}$ & 26 & 15.5 & 4 & & & 1 \\
\hline 6 & $\begin{array}{l}\text { Base de El Mogote, } \\
\text { camino a Sta. Agueda }\end{array}$ & 72 & 42.9 & 4 & & & \\
\hline 7 & Loma El Mogote & 14 & 8.3 & 2 & 1 & & 1 \\
\hline 8 & El Pilón de Najasa & 68 & 40.5 & 3 & & & 1 \\
\hline 9 & Cima El Pilón de Najasa & 11 & 6.6 & & & & \\
\hline 10 & Desfiladero de los Helechos & 4 & 2.3 & & 1 & & \\
\hline 11 & Orange & 58 & 34.5 & 2 & & & \\
\hline 12 & $\begin{array}{l}\text { Camino bajada de la } \\
\text { Sierra de Najasa al Cacaota }\end{array}$ & tal & 9.0 & 1 & 1 & & 1 \\
\hline
\end{tabular}


parcelas de $20 \times 20 \mathrm{~m}^{2}$ para las formaciones boscosas, de $10 \times 10 \mathrm{~m}^{2}$ para las sabanas antrópicas y de $4 \times 4 \mathrm{~m}^{2}$ para el complejo de vegetación del farallón o escarpa.

Los materiales se herborizaron y procesaron mediante las técnicas tradicionales; se determinaron y pasaron a formar parte de las colecciones del herbario del Centro de Investigaciones de Medio Ambiente de Camagüey (HACC).

Se listaron las especies muestreadas y se complementó la información relacionando el nombre común, tipo de endemismo, formaciones vegetales en que se establecen, utilidad económica y localidades trabajadas.

Las formaciones vegetales se consideraron de acuerdo a lo descrito por Pérez et al. (1994) para el complejo orográfico NajasaGuaicanámar-Cerro Cachimbo, y el tipo de endemismo y la relación florística de cada taxon según Borhidi (1976).

El análisis de las potencialidades como recurso natural se realizó a partir de Fors (1957), Havard-Duclos (1969), Roig (1974), Ordext (1978), EE.UU. (1979), Flores et al. (1988), Fuentes (1988), Hernández \& López (1991), y Arias (1994).

Se utilizó la cartografía digital para el procesamiento de la información sobre las formaciones vegetales y la confección del mapa de vegetación a escala 1:36 000. La información cartográfica básica utilizada se tomó de las hojas 1:10 000 del Instituto Cubano de Geodesia y Cartografía, actualizadas, correspondiente al área bajo estudio.

\section{RESULTADOS Y DISCUSIÓN}

La flora de la Sierra de Najasa se presenta en la Tabla 2. El $2.5 \%$ de los representantes de las plantas vasculares cubanas se encontraron en el área estudiada: 132 géneros y 167 especies pertenecientes a 64 familias. Fabaceae fue la familia botánica con mayor número de especies (19), seguida por Euphorbiaceae y Bromeliaceae con 5 y 7 , respectivamente.

La Figura 1 muestra el mapa de vegetación y las estaciones seleccionadas para el análisis del recurso florístico.

La riqueza de especies por estación y su representatividad con respecto a la flora del área se recogen en la Tabla 1. Las estaciones 6,8 y 11 poseen los valores más altos, y se caracterizan fisionómicamente por el bosque semideciduo notófilo y el bosque arbustoso, que forma parte de las comunidades de sustitución o reemplazo señaladas por Pérez et al. (1994). El valor más bajo la presentó la No. 2, monotípica, con la especie arbórea Oxandra lanceolata (Sw.) Baill. cuyo nombre común "Yaya" se utiliza para denominar estas poblaciones que generalmente componen uno de los estratos de la vegetación de complejo de mogote conocido como «Yayal». La No. 10 mostró también valores bajos lo cual responde a la estructura geomorfológica que la caracteriza y que permite, esencialmente, el desarrollo de pocas especies de helechos.

Los endémicos cubanos colectados representan el 8.6\% respecto a la flora del área, con 9 endemismos pancubanos, dos de Cuba Central-Cuba Oriental, uno de Cuba Central y uno de Cuba Occidental-Cuba Central (Tabla 2). Pérez et al. (1994) citaron 15 pancubanos, de los cuales cinco se hallaron en la Sierra donde a la vez tienen su hábitat Harrisia eriophora (Pfeiff.) Britt., Diospyros grisebachii (Hiern.) Standl., Platygyne hexandra (Jacq.) Muell. Arg., Hyperbaena racemosa Urb. y Passiflora cubensis L., no listados en ese trabajo, al igual que Malpighia suberosa Small y Piper aduncum L. ssp. ossanum (C. DC.) Trel., endemismos de Cuba Central-Cuba Oriental y de Cuba OccidentalCuba Central, respectivamente.

La localidad No. 1 (Cima del Farallón Colorado) posee el número mayor de endemismos pancubanos y en la No. 7 (Base de la loma "El Mogote") se halló la más alta riqueza de endemismos (Tabla 1), en las formaciones de bosque semideciduo mesófilo y notófilo. La distribución más amplia la presentaron total de 


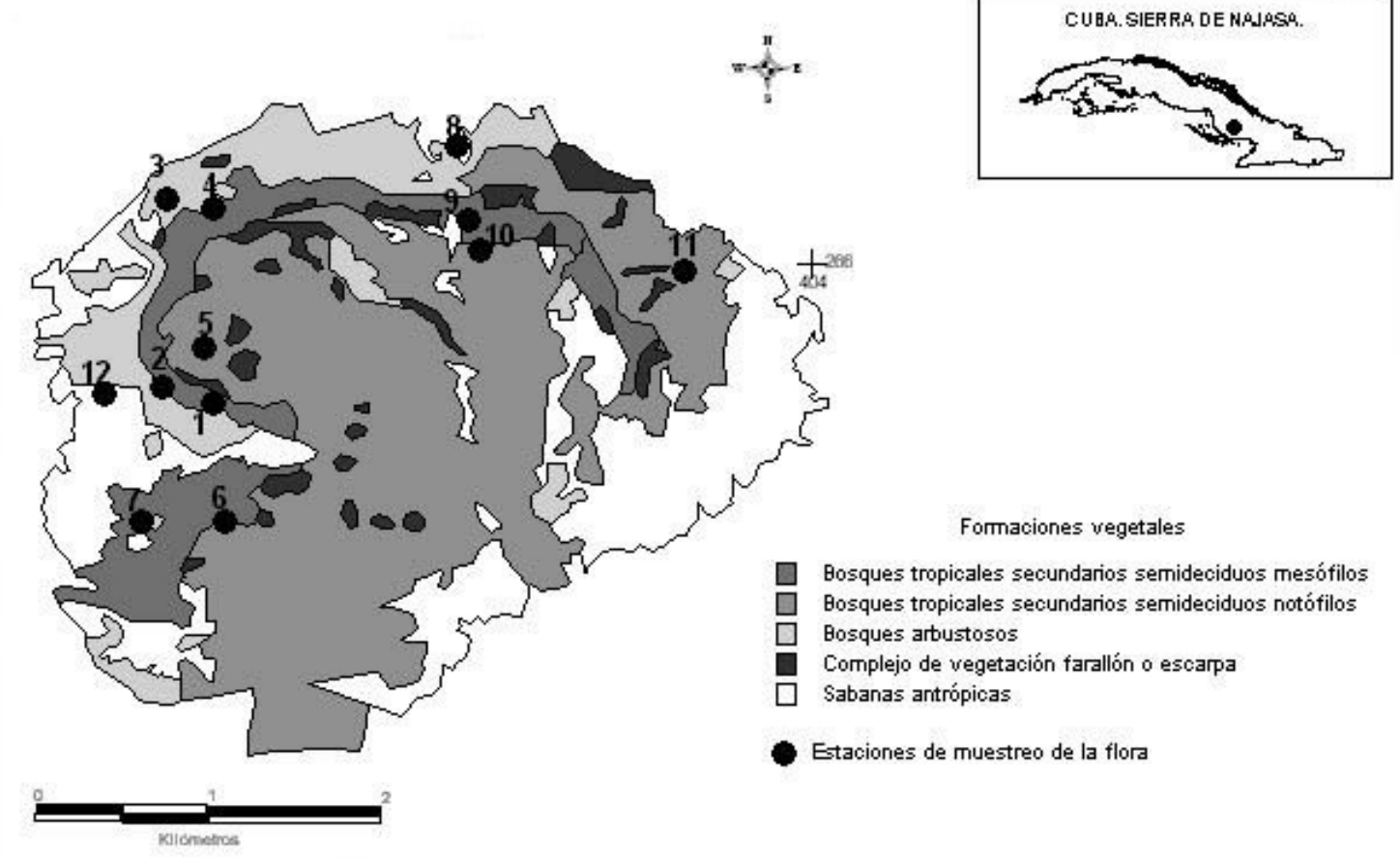

Figura 1 - Sierra Najasa: Vegetación.

Diospyros grisebachii (Hiern.) Standl. y Distictis gnaphalantha (A. Rich.) Urb. y, los de hábitats más restringidos Agave legrelliana Jacobi, Harrisia eriophora (Pfeiff.) Britt., Croton sagraeanus Muell. Arg. e Hyperbaena racemosa Urb. (Tabla 2 ).

La flora de la Sierra de Najasa está estrechamente relacionada con la del neotrópico; 54 especies son neotropicales (32.8\%). El $22.5 \%$ es afín a la del Caribe (36 especies) y el $12.2 \%$ a la de Las Antillas (30); el resto son elementos pantropicales (21). Un orden igual de afinidad refirieron Pérez et al. (1994) para la flora de las otras alturas del complejo orográfico.

La riqueza florística debe valorarse, además, desde el punto de vista económico por las potencialidades que como recurso natural le ofrece al hombre. La principal utilidad de sus componentes puede revertir en función de la salud humana, ya que 104 especies se reconocen como medicinales, 64 son melíferos, 47 maderables y 75 tienen otras aplicaciones.
Unas 128 especies, el 76.3\%, pueden ser aprovechadas social o económicamente. La Figura 2 recoge la utilidad de la flora por formaciones vegetales.

El Gobierno de la provincia aprobó en 1988 la categoría de Bosque Nacional para conservar los valores que esta área poseía, propuesta que se mantuvo por Pérez et al. (1994); en 1995, el Centro Nacional de Areas Protegidas (CNAP) asumió para el país las categorías de manejo de la UICN y se le reconoció como Paisaje Natural Protegido.

Los resultados obtenidos corroboran la necesidad de proteger la riqueza florística de la Sierra de Najasa y establecer un plan de manejo dentro de la planificación de los recursos del municipio en que se encuentra enmarcada, con el objetivo de controlar las afectaciones del entorno que pueden incidir sobre la pérdida de este valioso genofondo y en cuya conservación deben jugar un papel esencial las comunidades rurales vecinas como fuerza esencial para el desarrollo sostenible de la región. 


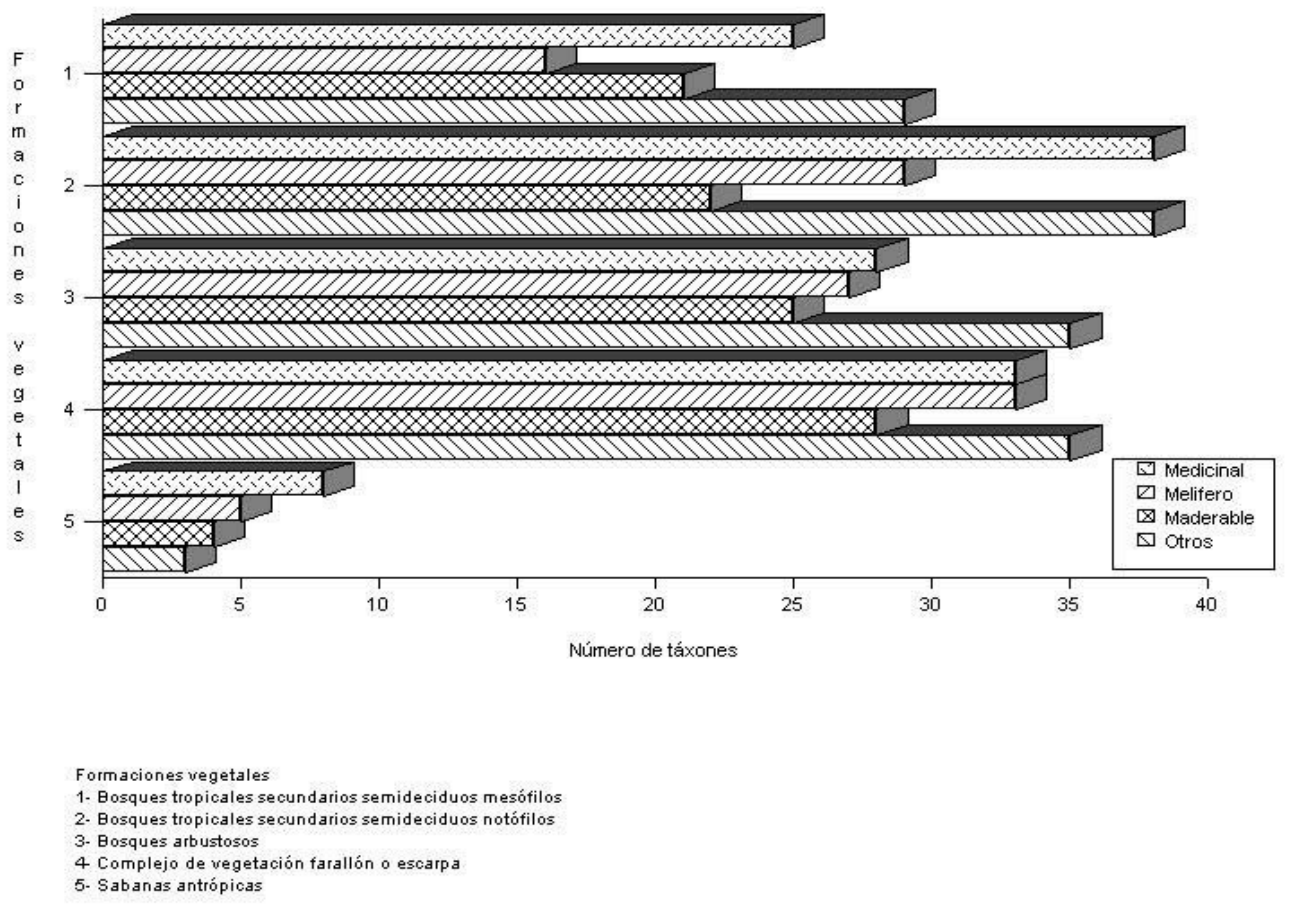

Figura 2 - Utilidad del recurso por formaciones vegetales.

Tabla 2 - Listado de los táxones de la Sierra de Najasa. Endemismo: PC, pancubano; COc-CC, Cuba Occidental-Cuba Central; CC-COr, Cuba Central-Cuba Oriental; CC, Cuba Central. Localidades 1 a la 12 se reflejan en la Tabla 1. Formaciones vegetales (F0R.VEG.): 1, bosques tropicales secundarios semideciduos mesófilos; 2, bosques tropicales secundarios semideciduos notófilos; 3 , bosques arbustosos; 4, complejo de vegetación farallón o escarpa; 5 , sabanas antrópicas. Usos: Med, medicinal; Mel, melífera; Mad, maderable; Otros, otras aplicaciones.

\begin{tabular}{|c|c|c|c|c|c|c|c|c|}
\hline \multirow[t]{2}{*}{ TAXON } & \multirow{2}{*}{$\begin{array}{l}\text { NOMBRE } \\
\text { COMUN }\end{array}$} & \multirow[t]{2}{*}{ ENDEMISMO } & \multicolumn{4}{|c|}{ USOS } & \multirow[t]{2}{*}{ LOCALIDAD } & \multirow{2}{*}{$\begin{array}{l}\text { FOR } \\
\text { VEG }\end{array}$} \\
\hline & & & MED & MAD & MEL & OTROS & & \\
\hline \multicolumn{9}{|l|}{ Acanthaceae } \\
\hline $\begin{array}{l}\text { Dicliptera } \\
\text { vahliana Nees }\end{array}$ & Gallitos & & $\mathrm{X}$ & & & & 6,11 & 2,4 \\
\hline $\begin{array}{l}\text { Thunbergia } \\
\text { alata Boj. ex Sims. }\end{array}$ & $\begin{array}{l}\text { Ojo } \\
\text { de Poeta }\end{array}$ & & & & & & 8,11 & 2,3 \\
\hline Agavaceae & & & & & & & & \\
\hline $\begin{array}{l}\text { Agave legrelliana } \\
\text { Jacobi }\end{array}$ & & $\mathrm{CC}$ & $\mathrm{X}$ & & & $\mathrm{X}$ & 4 & 1 \\
\hline Amaranthaceae & & & & & & & & \\
\hline $\begin{array}{l}\text { Alternanthera } \\
\text { sessilis (L.) R. } \\
\text { Br. ex DC. }\end{array}$ & Bella María & & & & & & $6,8,11$ & 2,3 \\
\hline
\end{tabular}




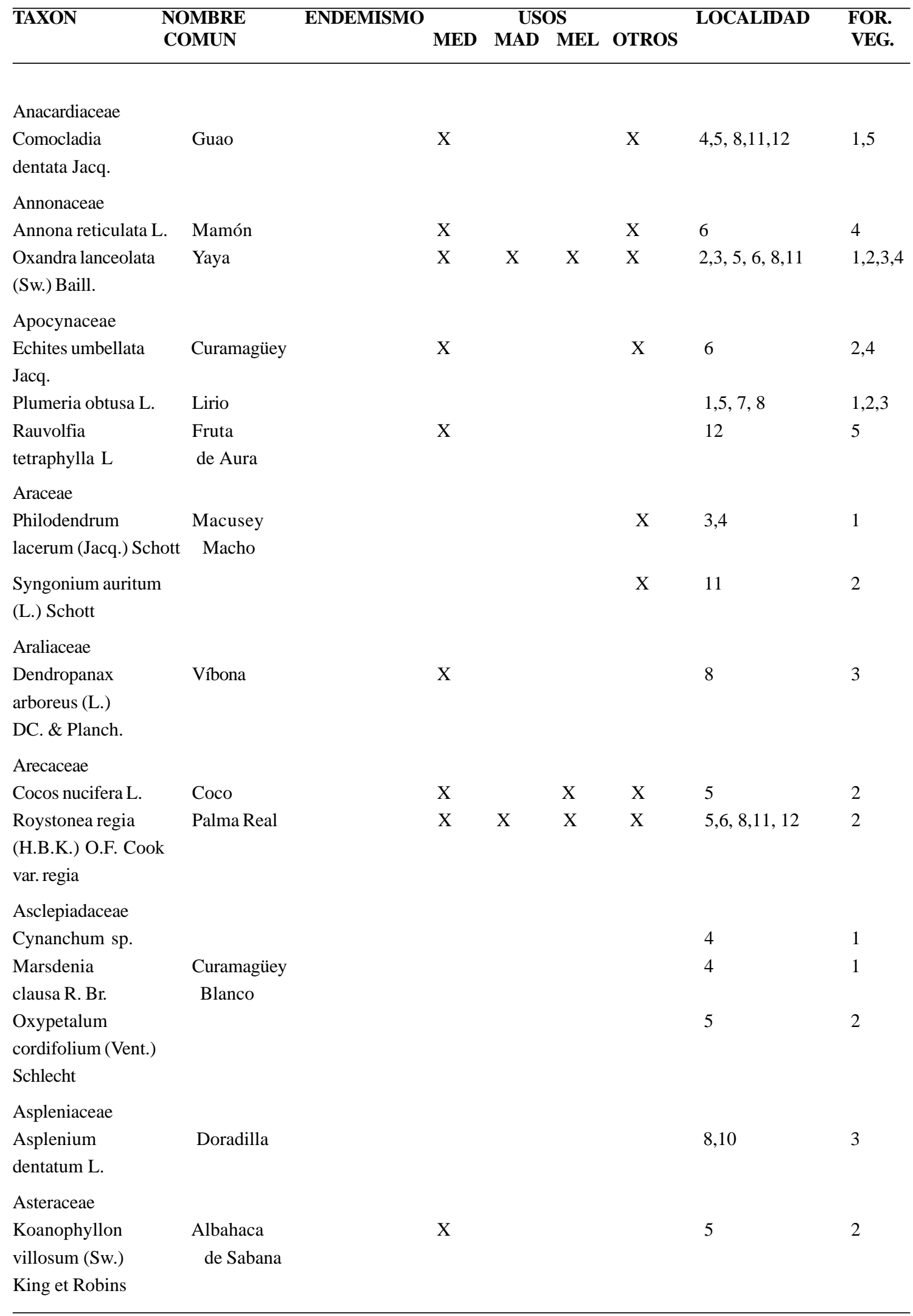


Aportes al conocimiento de la riqueza florística para la gestión ambiental de la Sierra de Najasa,

Camagüey, Cuba

\begin{tabular}{|c|c|c|c|c|c|c|c|c|}
\hline \multirow[t]{2}{*}{ TAXON } & \multirow{2}{*}{$\begin{array}{l}\text { JOMBRE } \\
\text { COMUN }\end{array}$} & \multirow[t]{2}{*}{ ENDEMISMO } & \multicolumn{4}{|c|}{ USOS } & \multirow[t]{2}{*}{ LOCALIDAD } & \multirow{2}{*}{$\begin{array}{l}\text { FOR. } \\
\text { VEG. }\end{array}$} \\
\hline & & & MED & MAD & MEL & OTROS & & \\
\hline $\begin{array}{l}\text { Mikania micrantha } \\
\text { H.B.K. }\end{array}$ & Guaco & & $\mathrm{X}$ & & & & 12 & 5 \\
\hline $\begin{array}{l}\text { Pluchea carolinensis } \\
\text { (Jacq.) G. Don }\end{array}$ & $\begin{array}{l}\text { Salvia } \\
\text { de Playa }\end{array}$ & & $\mathrm{X}$ & & $\mathrm{X}$ & & 6 & 2,4 \\
\hline $\begin{array}{l}\text { Pseudoelephantopus } \\
\text { spicatus (B. Juss. ex } \\
\text { Aubl.) C.F. Baker }\end{array}$ & $\begin{array}{l}\text { Lengua } \\
\text { de Vaca }\end{array}$ & & $\mathrm{X}$ & & & & $8,9,11,12$ & $1,2,3,5$ \\
\hline Trixis inula Crantz & $\begin{array}{l}\text { Palo } \\
\text { Santa María }\end{array}$ & & $\mathrm{X}$ & & & & $5,6,11$ & 2,4 \\
\hline $\begin{array}{l}\text { Vernonia menthifolia } \\
\text { (Poepp. ex Spreng.) Le }\end{array}$ & ess. & $\mathrm{PC}$ & $\mathrm{X}$ & & $\mathrm{X}$ & & $6,11,12$ & $2,4,5$ \\
\hline Bignoniaceae & & & & & & & & \\
\hline Crescentia cujete L. & Güira & & $\mathrm{X}$ & $\mathrm{X}$ & $\mathrm{X}$ & $X$ & 8 & 3 \\
\hline $\begin{array}{l}\text { Cydista diversifolia } \\
\text { (H.B.K.) Miers }\end{array}$ & $\begin{array}{l}\text { Bejuco } \\
\text { de Vieja }\end{array}$ & & & & $\mathrm{X}$ & & 11 & 2 \\
\hline $\begin{array}{l}\text { Distictis gnaphalantha } \\
\text { (A.Rich.) Urb. }\end{array}$ & & $\mathrm{PC}$ & & & & & $1,5,6,11$ & $1,2,4$ \\
\hline $\begin{array}{l}\text { Pithecoctenium } \\
\text { echinatum (Aubl.) } \\
\text { K. Schum }\end{array}$ & $\begin{array}{l}\text { Huevo de } \\
\text { Toro }\end{array}$ & & & & & & 6,8 & $2,3,4$ \\
\hline Bombacaceae & & & & & & & & \\
\hline $\begin{array}{l}\text { Ceiba pentandra } \\
\text { (L.) Gaertn. }\end{array}$ & Ceiba & & $\mathrm{X}$ & $\mathrm{X}$ & $X$ & $\mathrm{X}$ & 6,8 & 1 \\
\hline Boraginaceae & & & & & & & & \\
\hline Cordia collococca L. & Ateje & & & $\mathrm{X}$ & $\mathrm{X}$ & $\mathrm{X}$ & 6 & 2,4 \\
\hline $\begin{array}{l}\text { Cordia } \\
\text { gerascanthus L. }\end{array}$ & Varía & & $\mathrm{X}$ & $\mathrm{X}$ & $X$ & $\mathrm{X}$ & $4,6,7,8,9,11$ & $1,2,3,4$ \\
\hline Ehretia tinifolia L. & Roble Prieto & & & $\mathrm{X}$ & & $\mathrm{X}$ & 6 & 2,4 \\
\hline $\begin{array}{l}\text { Tournefortia } \\
\text { hirsutissima L. }\end{array}$ & Nigua & & $\mathrm{X}$ & & $\mathrm{X}$ & & 8 & 3 \\
\hline Bromeliaceae & & & & & & & & \\
\hline $\begin{array}{l}\text { Tillandsia argentea } \\
\text { Griseb. }\end{array}$ & & & & & & & 3,12 & 1,5 \\
\hline Tillandsia balbisiana & & & & & & & 11,12 & 5 \\
\hline Schult. & & & & & & & & \\
\hline Tillandsia festucoides & & & & & & & 5 & 2 \\
\hline Brogn. ex Mez & & & & & & & & \\
\hline Tillandsia recurvata L. & & & $\mathrm{X}$ & & & & 7 & 1 \\
\hline Tillandsia setacea Sw. & & & & & & & 5 & 2 \\
\hline Tillandsia usneoides L. & Guajaca & & $\mathrm{X}$ & & & $\mathrm{X}$ & 5 & 2 \\
\hline $\begin{array}{l}\text { Tillandsia } \\
\text { valenzuelana A. Rich. }\end{array}$ & & & & & & $\mathrm{X}$ & 3,5 & 1,2 \\
\hline
\end{tabular}




\begin{tabular}{|c|c|c|c|c|c|c|c|c|}
\hline \multirow[t]{2}{*}{ TAXON } & \multirow{2}{*}{$\begin{array}{l}\text { NOMBRE } \\
\text { COMUN }\end{array}$} & \multirow[t]{2}{*}{ ENDEMISMO } & \multicolumn{4}{|c|}{ USOS } & \multirow[t]{2}{*}{ LOCALIDAD } & \multirow{2}{*}{$\begin{array}{l}\text { FOR. } \\
\text { VEG. }\end{array}$} \\
\hline & & & MED & MAD & MEL & OTROS & & \\
\hline \multicolumn{9}{|l|}{ Burseraceae } \\
\hline $\begin{array}{l}\text { Bursera simaruba } \\
\text { (L.) Sargent. }\end{array}$ & Almácigo & & $\mathrm{X}$ & $\mathrm{X}$ & $\mathrm{X}$ & $\mathrm{X}$ & 5 & 2 \\
\hline \multicolumn{9}{|l|}{ Cactaceae } \\
\hline $\begin{array}{l}\text { Harrisia eriophora } \\
\text { (Pfeiff.) Britt. }\end{array}$ & Pitahaya & $\mathrm{PC}$ & & & & & 1 & 1 \\
\hline $\begin{array}{l}\text { Mamillaria prolifera } \\
\text { (Mill.) Haw. }\end{array}$ & & & & & & & 4 & 1 \\
\hline $\begin{array}{l}\text { Pilosocereus } \\
\text { grandiflorus (L.) }\end{array}$ & Jíjira & CC-COr & $X$ & & & & 4 & 1 \\
\hline Britt. et Rose & & & & & & & & \\
\hline $\begin{array}{l}\text { Selenicereus } \\
\text { grandiflorus (L.) } \\
\text { Britt. et Rose }\end{array}$ & Pitahaya & & $\mathrm{X}$ & & & $\mathrm{X}$ & $3,4,5$ & 1,2 \\
\hline \multicolumn{9}{|l|}{ Canellaceae } \\
\hline $\begin{array}{l}\text { Canella winterana } \\
\text { (L.) Gaertn. }\end{array}$ & Cúrbana & & $\mathrm{X}$ & $\mathrm{X}$ & $X$ & $\mathrm{X}$ & 8,10 & 3 \\
\hline \multicolumn{9}{|l|}{ Capparaceae } \\
\hline Capparis flexuosa L. & $\begin{array}{l}\text { Palo Barba } \\
\text { de Indio }\end{array}$ & & & $X$ & & & $1,4,7,12$ & 1,5 \\
\hline \multicolumn{9}{|l|}{ Cecropiaceae } \\
\hline $\begin{array}{l}\text { Cecropia peltata L. } \\
\text { Clusiaceae }\end{array}$ & Yagruma & & $X$ & $\mathrm{X}$ & & $X$ & 6,8 & $2,3,4$ \\
\hline $\begin{array}{l}\text { Calophyllum } \\
\text { antillanum Britt. }\end{array}$ & Ocuje & & $\mathrm{X}$ & $\mathrm{X}$ & $\mathrm{X}$ & $\mathrm{X}$ & $8,9,11$ & $1,2,3$ \\
\hline \multicolumn{9}{|l|}{ Convolvulaceae } \\
\hline $\begin{array}{l}\text { Ipomoea cf. nil } \\
\text { (L.) Roth }\end{array}$ & $\begin{array}{l}\text { Aguinaldo } \\
\text { Azul Claro }\end{array}$ & & & & & & 8 & 3 \\
\hline $\begin{array}{l}\text { Ipomoea } \\
\text { hederifolia L. }\end{array}$ & $\begin{array}{r}\text { Cambustera de } \\
\text { Hojas Anchas }\end{array}$ & & & & & & $6,8,11$ & $2,3,4$ \\
\hline $\begin{array}{l}\text { Turbina corymbosa } \\
\text { (L.) Raf. }\end{array}$ & $\begin{array}{l}\text { Aguinaldo } \\
\text { de Pascua }\end{array}$ & & $\mathrm{X}$ & & $\mathrm{X}$ & & 11 & 2 \\
\hline \multicolumn{9}{|l|}{ Cucurbitaceae } \\
\hline $\begin{array}{l}\text { Anguria pedata } \\
\text { (L.) Jacq. }\end{array}$ & $\begin{array}{l}\text { Pepino } \\
\text { cimarrón }\end{array}$ & & & & & & 8 & 3 \\
\hline $\begin{array}{l}\text { Momordica } \\
\text { charantia L. }\end{array}$ & Cundeamor & & $X$ & & $X$ & & 6,11 & 2,4 \\
\hline Dryopteridaceae & & & & & & & & \\
\hline $\begin{array}{l}\text { Cyclopeltis } \\
\text { semicordata } \\
\text { (Sw.) J. Smith }\end{array}$ & & & & & & & 10 & 2 \\
\hline
\end{tabular}


Aportes al conocimiento de la riqueza florística para la gestión ambiental de la Sierra de Najasa,

Camagüey, Cuba

\begin{tabular}{|c|c|c|c|c|c|c|c|c|}
\hline \multirow[t]{2}{*}{ TAXON } & \multirow{2}{*}{$\begin{array}{l}\text { OOMBRE } \\
\text { COMUN }\end{array}$} & \multirow{2}{*}{ ENDEMISMO } & \multicolumn{4}{|c|}{ USOS } & \multirow[t]{2}{*}{ LOCALIDAD } & \multirow{2}{*}{$\begin{array}{l}\text { FOR. } \\
\text { VEG. }\end{array}$} \\
\hline & & & MED & MAD & MEL & OTROS & & \\
\hline \multicolumn{9}{|l|}{ Ebenaceae } \\
\hline $\begin{array}{l}\text { Diospyros grisebachii } \\
\text { (Hiern.) Standl. }\end{array}$ & $\begin{array}{c}\text { Ebano } \\
\text { Real }\end{array}$ & $\mathrm{PC}$ & & $X$ & $\mathrm{X}$ & $\mathrm{X}$ & $1,3,4,5,7,8$ & $1,2,3$ \\
\hline $\begin{array}{l}\text { Erythroxylaceae } \\
\text { Erythroxylum } \\
\text { havanense Jacq. } \\
\text { ssp. havanense }\end{array}$ & Jibá & & $X$ & $\mathrm{X}$ & $\mathrm{X}$ & $\mathrm{X}$ & $1,3,6,11,12$ & $1,2,4,5$ \\
\hline Euphorbiaceae & & & & & & & & \\
\hline Adelia ricinella $\mathrm{L}$. & Jía & & & $\mathrm{X}$ & $X$ & $\mathrm{X}$ & $1,4,6,8$ & $1,2,3,4$ \\
\hline $\begin{array}{l}\text { Ateramnus lucidus } \\
\text { (Sw.) Rothm. }\end{array}$ & Yaití & & $\mathrm{X}$ & $\mathrm{X}$ & $\mathrm{X}$ & $\mathrm{X}$ & 1,7 & 1 \\
\hline Croton lucidus L. & Cuabilla & & & & & & 5,6 & 2,4 \\
\hline $\begin{array}{l}\text { Croton sagraeanus } \\
\text { Muell. Arg. }\end{array}$ & Aceitillo & $\mathrm{PC}$ & $X$ & & $\mathrm{X}$ & & 1 & 1 \\
\hline $\begin{array}{l}\text { Euphorbia heterophylla } \\
\text { L. var. heterophylla }\end{array}$ & & & $X$ & & $X$ & & 6 & 2,4 \\
\hline $\begin{array}{l}\text { Platygyne hexandra } \\
\text { (Jacq.) Muell. Arg. }\end{array}$ & Ortiguilla & $\mathrm{PC}$ & $\mathrm{X}$ & & & $\mathrm{X}$ & $5,6,8$ & $2,3,4$ \\
\hline $\begin{array}{l}\text { Tragia volubilis L. } \\
\text { Fabaceae- } \\
\text { Caesalpinioideae }\end{array}$ & Candelilla & & $X$ & & & & 11 & 2 \\
\hline $\begin{array}{l}\text { Poeppigia procera } \\
\text { Presl. }\end{array}$ & Tengue & & $\mathrm{X}$ & $\mathrm{X}$ & & $\mathrm{X}$ & $1,6,8,9,11$ & $1,2,3,4$ \\
\hline $\begin{array}{l}\text { Senna spectabilis } \\
\text { (DC.) Irwin et } \\
\text { Barneby var. } \\
\text { spectabilis }\end{array}$ & Algarrobillo & & & $\mathrm{X}$ & & $\mathrm{X}$ & $6,11,1,4$ & $1,2,4$ \\
\hline Fabaceae-Faboideae & & & & & & & & \\
\hline $\begin{array}{l}\text { Ateleia cubensis } \\
\text { Griseb. var. cubensis }\end{array}$ & $\begin{array}{l}\text { Rala de } \\
\text { Gallina }\end{array}$ & & & & & & $6,8,11$ & $2,3,4$ \\
\hline $\begin{array}{l}\text { Calopogonium } \\
\text { coeruleum } \\
\text { (Benth.) Hemsl. }\end{array}$ & $\begin{array}{l}\text { Jícama } \\
\text { Dulce }\end{array}$ & & & & $\mathrm{x}$ & & $5,6,8,11,12$ & $2,3,4,5$ \\
\hline $\begin{array}{l}\text { Canavalia } \\
\text { rosea (Sw.) DC. }\end{array}$ & $\begin{array}{l}\text { Mate } \\
\text { Colorado }\end{array}$ & $\mathrm{PC}$ & $X$ & & & $\mathrm{X}$ & $5,7,8$ & $1,2,3$ \\
\hline $\begin{array}{l}\text { Centrosema plumieri } \\
\text { (Turp. et Pers.) Benth. }\end{array}$ & & & $\mathrm{X}$ & & & $X$ & 11 & 2 \\
\hline $\begin{array}{l}\text { Centrosema } \\
\text { pubescens Benth }\end{array}$ & $\begin{array}{l}\text { Bejuco } \\
\text { de Chivo }\end{array}$ & & & & & $\mathrm{X}$ & 6,11 & 2,4 \\
\hline $\begin{array}{l}\text { Centrosema } \\
\text { virginianum (L.) Benth. }\end{array}$ & Azulada & & $\mathrm{X}$ & & $\mathrm{X}$ & $\mathrm{X}$ & 6 & 2,4 \\
\hline Crotalaria incana L. & Garbancillo & & & & & $\mathrm{X}$ & 6 & 2,4 \\
\hline $\begin{array}{l}\text { Desmodium incanum } \\
\text { DC. var. incanum }\end{array}$ & Amor Seco & & $\mathrm{X}$ & & & & 6 & $2,3,4$ \\
\hline
\end{tabular}

Rodriguésia 53 (82): 131-145. 2002 


\begin{tabular}{|c|c|c|c|c|c|c|c|c|}
\hline TAXON & $\begin{array}{l}\text { OMBRE } \\
\text { OMUN }\end{array}$ & ENDEMISMO & \multicolumn{4}{|c|}{ USOS } & LOCALIDAD & $\begin{array}{l}\text { FOR. } \\
\text { VEG. }\end{array}$ \\
\hline $\begin{array}{l}\text { Desmodium } \\
\text { scorpiurus (Sw.) Desv. }\end{array}$ & & & & & & & 6 & 2,4 \\
\hline $\begin{array}{l}\text { Hebestigma cubense } \\
\text { (H.B.K.) Urb. }\end{array}$ & Frijolillo & & & $\mathrm{X}$ & $\mathrm{X}$ & & $1,6,8,12$ & $\begin{array}{l}1,2,3 \\
4,5\end{array}$ \\
\hline $\begin{array}{l}\text { Fabaceae- } \\
\text { Mimosoideae }\end{array}$ & & & & & & & & \\
\hline $\begin{array}{l}\text { Abarema glauca } \\
\text { (Urb.) Barneby } \\
\text { et J. W. Grimes }\end{array}$ & & & & & $\mathrm{X}$ & & 8 & 3 \\
\hline $\begin{array}{l}\text { Acacia farnesiana } \\
\text { (L.) Willd. }\end{array}$ & $\begin{array}{l}\text { Aroma } \\
\text { Amarilla }\end{array}$ & & $\mathrm{X}$ & & $\mathrm{X}$ & $\mathrm{X}$ & 6 & 2,4 \\
\hline $\begin{array}{l}\text { Acacia tenuifolia } \\
\text { (L.) Willd. }\end{array}$ & Tocino & & & & & & $3,4,6$ & $1,2,4$ \\
\hline $\begin{array}{l}\text { Desmanthus } \\
\text { virgatus (L.) Willd. }\end{array}$ & Adormidera & & & & & & 1 & 1 \\
\hline $\begin{array}{l}\text { Lysiloma sabicu } \\
\text { Benth. }\end{array}$ & Sabicú & & & $\mathrm{X}$ & & & 6 & 2,4 \\
\hline $\begin{array}{l}\text { Samanea saman } \\
\text { (Jacq.) Merr. }\end{array}$ & Algarrobo & & $\mathrm{X}$ & $X$ & $\mathrm{X}$ & $\mathrm{X}$ & 6,8 & $2,3,4$ \\
\hline $\begin{array}{l}\text { Zapoteca formosa } \\
\text { (Kunth) H.M. Hern. } \\
\text { ssp. formosa }\end{array}$ & & & & $\mathrm{X}$ & $\mathrm{X}$ & $\mathrm{X}$ & 6,8 & $2,3,4$ \\
\hline Flacourtiaceae & & & & & & & & \\
\hline $\begin{array}{l}\text { Casearia aculeata } \\
\text { Jacq. }\end{array}$ & Jía Brava & & $\mathrm{X}$ & $\mathrm{X}$ & $\mathrm{X}$ & & 6,11 & 2,4 \\
\hline $\begin{array}{l}\text { Casearia guianensis } \\
\text { (Aubl.) Urb. }\end{array}$ & Jía Amarilla & & & $\mathrm{X}$ & & & 6 & 2,4 \\
\hline Lamiaceae & & & & & & & & \\
\hline $\begin{array}{l}\text { Hyptis pectinata } \\
\text { (L.) Poit. }\end{array}$ & Alhucema & & $\mathrm{X}$ & & $\mathrm{X}$ & & 11 & 2 \\
\hline Lauraceae & & & & & & & & \\
\hline $\begin{array}{l}\text { Licaria triandria } \\
\text { (Sw.) Kosterm. }\end{array}$ & Leviza & & & $\mathrm{X}$ & $\mathrm{X}$ & $\mathrm{X}$ & 8 & 3 \\
\hline $\begin{array}{l}\text { Nectandra coriacea } \\
\text { (Sw.) Griseb. }\end{array}$ & Cigua & & & $\mathrm{X}$ & & $\mathrm{X}$ & $5,6,8,11$ & $2,3,4$ \\
\hline Malpighiaceae & & & & & & & & \\
\hline $\begin{array}{l}\text { Bunchosia } \\
\text { swartziana Griseb. }\end{array}$ & & & & & & & 7,8 & 1,3 \\
\hline $\begin{array}{l}\text { Malpighia } \\
\text { suberosa Small }\end{array}$ & $\begin{array}{l}\text { Palo } \\
\text { Bronco }\end{array}$ & CC-COr & & & & & 6 & 2,4 \\
\hline $\begin{array}{l}\text { Stigmaphyllon } \\
\text { diversifolium } \\
\text { (Kunth) Juss. }\end{array}$ & $\begin{array}{l}\text { Bejuco } \\
\text { Blanco }\end{array}$ & & & & & & 8,11 & 2,3 \\
\hline
\end{tabular}


Aportes al conocimiento de la riqueza florística para la gestión ambiental de la Sierra de Najasa,

Camagüey, Cuba

\begin{tabular}{|c|c|c|c|c|c|c|c|c|}
\hline \multirow[t]{2}{*}{ TAXON } & \multirow{2}{*}{$\begin{array}{l}\text { NOMBRE } \\
\text { COMUN }\end{array}$} & \multirow{2}{*}{ ENDEMISMO } & \multicolumn{4}{|c|}{ USOS } & \multirow[t]{2}{*}{ LOCALIDAD } & \multirow{2}{*}{$\begin{array}{l}\text { FOR. } \\
\text { VEG. }\end{array}$} \\
\hline & & & MED & MAD & MEL & OTROS & & \\
\hline \multicolumn{9}{|l|}{ Malvaceae } \\
\hline $\begin{array}{l}\text { Pavonia fruticosa } \\
\text { (Mill.) Fawc. et Rendle }\end{array}$ & Tábano & & $\mathrm{X}$ & & & & 8 & 3 \\
\hline $\begin{array}{l}\text { Pavonia spinifex } \\
\text { (L.) Cav. }\end{array}$ & $\begin{array}{l}\text { Majagüilla } \\
\text { de Costa }\end{array}$ & & $\mathrm{X}$ & & & & 6,11 & 2,4 \\
\hline Sida glutinosa Cav. & $\begin{array}{l}\text { Malva } \\
\text { de Cuba }\end{array}$ & & $\mathrm{X}$ & & $\mathrm{X}$ & & 6 & 2,4 \\
\hline Sida pyramidata Cav. & & & & & $\mathrm{X}$ & & 8 & 3 \\
\hline Sida rhombifolia L. & $\begin{array}{l}\text { Malva } \\
\text { de Cochino }\end{array}$ & & $\mathrm{X}$ & & $\mathrm{x}$ & & 6 & 2,4 \\
\hline Sida spinosa L. & & & & & $\mathrm{X}$ & $\mathrm{X}$ & 11 & 2 \\
\hline \multicolumn{9}{|l|}{ Meliaceae } \\
\hline Cedrela odorata L. & Cedro & & $\mathrm{X}$ & $\mathrm{X}$ & $\mathrm{X}$ & $\mathrm{X}$ & 4,6 & $1,2,4$ \\
\hline $\begin{array}{l}\text { Guarea guidonia } \\
\text { (L.) Sleumer }\end{array}$ & Yamagua & & $\mathrm{X}$ & $\mathrm{X}$ & $\mathrm{X}$ & $\mathrm{X}$ & 6,11 & 2,4 \\
\hline $\begin{array}{l}\text { Swietenia } \\
\text { mahagoni (L.) Jacq. }\end{array}$ & $\begin{array}{l}\text { Caoba } \\
\text { de Cuba }\end{array}$ & & $\mathrm{X}$ & $\mathrm{X}$ & $\mathrm{X}$ & $\mathrm{X}$ & 6 & 2,4 \\
\hline $\begin{array}{l}\text { Trichilia } \\
\text { havanensis Jacq. }\end{array}$ & Siguaraya & & $\mathrm{X}$ & $\mathrm{X}$ & $\mathrm{X}$ & $\mathrm{X}$ & $6,8,9,11$ & $1,2,3,4$ \\
\hline Trichilia hirta L. & $\begin{array}{l}\text { Cabo } \\
\text { de Hacha }\end{array}$ & & & $\mathrm{X}$ & $\mathrm{X}$ & $\mathrm{X}$ & 6 & 2,4 \\
\hline \multicolumn{9}{|l|}{ Menispermaceae } \\
\hline $\begin{array}{l}\text { Hyperbaena } \\
\text { racemosa Urb. }\end{array}$ & Chicharrón & $\mathrm{PC}$ & & & & & 6 & 2,4 \\
\hline \multicolumn{9}{|l|}{ Moraceae } \\
\hline Ficus aurea Nutt. & $\begin{array}{l}\text { Jagüey } \\
\text { Hembra }\end{array}$ & & & $\mathrm{X}$ & & & 7,8 & 1,3 \\
\hline \multicolumn{9}{|l|}{ Myrtaceae } \\
\hline $\begin{array}{l}\text { Eugenia } \\
\text { maleolens Poir. }\end{array}$ & & & & $\mathrm{X}$ & & & 8 & 3 \\
\hline $\begin{array}{l}\text { Myrciaria floribunda } \\
\text { (West.ex Willd.) Berg. }\end{array}$ & Mije & & & $\mathrm{X}$ & & $\mathrm{X}$ & 8 & 3 \\
\hline Nyctaginaceae & & & & & & & & \\
\hline Pisonia aculeata L. & Zarza & & $\mathrm{X}$ & $\mathrm{X}$ & $\mathrm{X}$ & $\mathrm{X}$ & 11 & 2 \\
\hline Orchidaceae & & & & & & & & \\
\hline $\begin{array}{l}\text { Oeceoclades maculata } \\
\text { (Ldl.)Ldl. }\end{array}$ & & & & & & & $6,8,11$ & 2,3 \\
\hline $\begin{array}{l}\text { Vanilla phaeantha } \\
\text { Rchb. f. }\end{array}$ & & & $\mathrm{X}$ & & & & 5 & 2 \\
\hline Passifloraceae & & & & & & & & \\
\hline Passiflora capsularis L. & & & & & $\mathrm{X}$ & & 6,11 & 2,4 \\
\hline $\begin{array}{l}\text { Passiflora } \\
\text { cubensis L. }\end{array}$ & $\begin{array}{l}\text { Gürito } \\
\text { de Pasión }\end{array}$ & $\mathrm{PC}$ & $\mathrm{X}$ & & $\mathrm{X}$ & $\mathrm{X}$ & 1,4 & 1 \\
\hline
\end{tabular}

Rodriguésia 53 (82): 131-145. 2002 


\begin{tabular}{|c|c|c|c|c|c|c|c|c|}
\hline \multirow[t]{2}{*}{ TAXON } & \multirow{2}{*}{$\begin{array}{l}\text { NOMBRE } \\
\text { COMUN }\end{array}$} & \multirow{2}{*}{ ENDEMISMO } & \multicolumn{4}{|c|}{ USOS } & \multirow[t]{2}{*}{ LOCALIDAD } & \multirow{2}{*}{$\begin{array}{l}\text { FOR. } \\
\text { VEG. }\end{array}$} \\
\hline & & & MED & MAD & MEL & OTROS & & \\
\hline $\begin{array}{l}\text { Passiflora } \\
\text { suberosa L. }\end{array}$ & $\begin{array}{l}\text { Huevo } \\
\text { de Gallo }\end{array}$ & & & & $\mathrm{X}$ & & $5,6,11$ & 2,4 \\
\hline \multicolumn{9}{|l|}{ Phytolacaceae } \\
\hline Rivina humilis L. & Coralitos & & $\mathrm{X}$ & & $\mathrm{X}$ & $\mathrm{X}$ & 8 & 3 \\
\hline $\begin{array}{l}\text { Trichostigma } \\
\text { octandrum (L.) H.Walt. }\end{array}$ & $\begin{array}{l}\text { Bejuco } \\
\text { lt. Canasta }\end{array}$ & & & & & $\mathrm{X}$ & 9 & 1 \\
\hline Picramniaceae & & & & & & & & \\
\hline $\begin{array}{l}\text { Picramnia } \\
\text { pentandra Sw. }\end{array}$ & Aguedita & & $\mathrm{X}$ & $\mathrm{X}$ & $\mathrm{X}$ & $\mathrm{X}$ & 8 & 3 \\
\hline \multicolumn{9}{|l|}{ Piperaceae } \\
\hline $\begin{array}{l}\text { Piper aduncum L. ssp. } \\
\text { ossanum (C.DC.) Trel. }\end{array}$ & $\begin{array}{l}\text { Platanillo } \\
\text { 1. de Cuba }\end{array}$ & $\mathrm{COc}-\mathrm{CC}$ & $X$ & & & & $6,9,11$ & $1,2,4$ \\
\hline Piper amalago L. & & & & & & $\mathrm{X}$ & $8,9,11$ & $1,2,3$ \\
\hline Piper peltatum L. & Caisimón & & & & & & 8 & 3 \\
\hline Plumbaginaceae & & & & & & & & \\
\hline Plumbago scandens L. & Malacara & & $\mathrm{X}$ & & & & 11,12 & 2,5 \\
\hline Poaceae & & & & & & & & \\
\hline $\begin{array}{l}\text { Lasiacis divaricata } \\
\text { (L.) Hitchc. }\end{array}$ & $\begin{array}{l}\text { Pitillo } \\
\text { de Monte }\end{array}$ & & $X$ & & & $\mathrm{X}$ & $1,5,6,11$ & $1,2,4$ \\
\hline Olyra latifolia L. & Tibisí & & $\mathrm{X}$ & & & $\mathrm{X}$ & $8,9,11$ & $1,2,3$ \\
\hline Polipodiaceae & & & & & & & & \\
\hline $\begin{array}{l}\text { Polipodium } \\
\text { heterophyllum L. }\end{array}$ & & & & & & & 8 & 3 \\
\hline Polygalaceae & & & & & & & & \\
\hline $\begin{array}{l}\text { Securidaca lamarckii } \\
\text { Griseb. }\end{array}$ & $\begin{array}{l}\text { Flor de } \\
\text { la Cruz }\end{array}$ & & & & & & 12 & 5 \\
\hline Pteridaceae & & & & & & & & \\
\hline $\begin{array}{l}\text { Adiantum } \\
\text { melanoleucum Willd. }\end{array}$ & & & & & & & $8,10,11$ & 2,3 \\
\hline $\begin{array}{l}\text { Adiantum pyramidale } \\
\text { (L.) Willd. }\end{array}$ & & & & & & & 6,11 & 2,4 \\
\hline $\begin{array}{l}\text { Cheylanthes } \\
\text { microphylla(Sw.) Sw. }\end{array}$ & & & & & & & 1 & 1 \\
\hline Rhamnaceae & & & & & & & & \\
\hline $\begin{array}{l}\text { Colubrina elliptica } \\
\text { (Sw.) Brizicki et Stern }\end{array}$ & Jayabico & & $\mathrm{X}$ & $\mathrm{X}$ & & $X$ & 7,8 & 1,3 \\
\hline $\begin{array}{l}\text { Gouania lupuloides } \\
\text { (L.) Urb. var. } \\
\text { lupuloides }\end{array}$ & Jaboncillo & & $\mathrm{X}$ & & & $\mathrm{X}$ & 6,8 & $2,3,4$ \\
\hline $\begin{array}{l}\text { Gouania polygama } \\
\text { (Jacq.) Urb. }\end{array}$ & Jaboncillo & & $\mathrm{X}$ & & $X$ & $\mathrm{X}$ & 6 & 2,4 \\
\hline
\end{tabular}


Aportes al conocimiento de la riqueza florística para la gestión ambiental de la Sierra de Najasa,

Camagüey, Cuba

\begin{tabular}{|c|c|c|c|c|c|c|c|}
\hline \multirow[t]{2}{*}{ TAXON } & \multirow{2}{*}{$\begin{array}{l}\text { NOMBRE } \\
\text { COMUN }\end{array}$} & \multirow[t]{2}{*}{ ENDEMISMO } & \multicolumn{3}{|c|}{ USOS } & \multirow[t]{2}{*}{ LOCALIDAD } & \multirow{2}{*}{$\begin{array}{l}\text { FOR. } \\
\text { VEG. }\end{array}$} \\
\hline & & & MAD & MEL & OTROS & & \\
\hline \multicolumn{8}{|l|}{ Rubiaceae } \\
\hline $\begin{array}{l}\text { Borreria laevis } \\
\text { (L.) C. et S. }\end{array}$ & $\begin{array}{l}\text { Hierba } \\
\text { de Garro }\end{array}$ & & & & & 6 & 2,4 \\
\hline $\begin{array}{l}\text { Chiococca alba } \\
\text { (L.) Hitchc }\end{array}$ & $\begin{array}{l}\text { Bejuco } \\
\text { de Verraco }\end{array}$ & $X$ & & & & 8 & 3 \\
\hline $\begin{array}{l}\text { Hamelia } \\
\text { patens Jacq. }\end{array}$ & Ponasí & $X$ & & $\mathrm{X}$ & $\mathrm{X}$ & 9 & 1 \\
\hline \multicolumn{8}{|l|}{ Rutaceae } \\
\hline $\begin{array}{l}\text { Amyris } \\
\text { elemifera L. }\end{array}$ & $\begin{array}{l}\text { Cuaba } \\
\text { Amarilla } \\
\text { de Costa }\end{array}$ & $X$ & & & & 7 & 1 \\
\hline $\begin{array}{l}\text { Citrus limon } \\
\text { (L.) Burm.f. }\end{array}$ & Limón & $\mathrm{X}$ & $X$ & $\mathrm{X}$ & $\mathrm{X}$ & 8,11 & 2,3 \\
\hline $\begin{array}{l}\text { Citrus sinensis } \\
\text { (L.) Osbeck }\end{array}$ & $\begin{array}{c}\text { Naranja } \\
\text { Dulce }\end{array}$ & $\mathrm{X}$ & & & $X$ & 11 & 2 \\
\hline $\begin{array}{l}\text { Zanthoxylum } \\
\text { martinicense (Lam.) DC }\end{array}$ & $\begin{array}{l}\text { Ayúa } \\
\text { DC. }\end{array}$ & $X$ & $X$ & $X$ & $\mathrm{X}$ & $5,6,8,11$ & $2,3,4$ \\
\hline \multicolumn{8}{|l|}{ Sapindaceae } \\
\hline Cupania americana L. & $\begin{array}{l}\text { Guara } \\
\text { común }\end{array}$ & $X$ & $X$ & $X$ & $X$ & 6,11 & 2,4 \\
\hline $\begin{array}{l}\text { Cupania glabra } \\
\text { Sw. var. glabra }\end{array}$ & $\begin{array}{l}\text { Guara } \\
\text { de Costa }\end{array}$ & & $X$ & $X$ & $X$ & $5,7,8,11$ & $1,2,3$ \\
\hline $\begin{array}{l}\text { Melicoccus } \\
\text { bijugatus Jacq. }\end{array}$ & Mamoncillo & $\mathrm{X}$ & $X$ & $\mathrm{X}$ & $\mathrm{X}$ & 11 & 2 \\
\hline $\begin{array}{l}\text { Paullinia } \\
\text { fuscescens H.B.K. }\end{array}$ & $\begin{array}{l}\text { Bejuco } \\
\text { de Vieja }\end{array}$ & & & & $\mathrm{X}$ & $6,8,11$ & $2,3,4$ \\
\hline $\begin{array}{l}\text { Paullinia } \\
\text { jamaicensis Macf. }\end{array}$ & $\begin{array}{l}\text { Bejuco } \\
\text { Matancero }\end{array}$ & & & & & 8 & 3 \\
\hline \multicolumn{8}{|l|}{ Sapotaceae } \\
\hline $\begin{array}{l}\text { Chrysophyllum } \\
\text { oliviforme L. }\end{array}$ & Caimitillo & & $\mathrm{X}$ & $\mathrm{X}$ & $X$ & 6,11 & 2,4 \\
\hline $\begin{array}{l}\text { Sideroxylon } \\
\text { foetidissimum Jacq. } \\
\text { ssp. foetidissimum }\end{array}$ & Jocúma & $\mathrm{X}$ & $\mathrm{X}$ & & $\mathrm{X}$ & $6,7,8$ & $1,2,3,4$ \\
\hline Schizaeaceae & & & & & & & \\
\hline $\begin{array}{l}\text { Anemia adiantifiolia } \\
\text { (L.) Sw. }\end{array}$ & & & & & & 6,11 & 2,4 \\
\hline Simaroubaceae & & & & & & & \\
\hline $\begin{array}{l}\text { Simarouba glauca DC. } \\
\text { var. typica Cronquist }\end{array}$ & Gavilán & $\mathrm{X}$ & & $\mathrm{X}$ & & 11 & 2 \\
\hline Smilacaceae & & & & & & & \\
\hline $\begin{array}{l}\text { Smilax domingensis } \\
\text { Willd. }\end{array}$ & $\begin{array}{r}\text { Raíz de } \\
\text { China }\end{array}$ & & & $\mathrm{X}$ & $\mathrm{X}$ & 8 & 3 \\
\hline
\end{tabular}




\begin{tabular}{|c|c|c|c|c|c|c|c|c|}
\hline \multirow[t]{2}{*}{ TAXON } & \multirow{2}{*}{$\begin{array}{l}\text { NOMBRE } \\
\text { COMUN }\end{array}$} & \multirow{2}{*}{ ENDEMISMO } & \multicolumn{4}{|c|}{ USOS } & \multirow[t]{2}{*}{ LOCALIDAD } & \multirow{2}{*}{$\begin{array}{l}\text { FOR. } \\
\text { VEG. }\end{array}$} \\
\hline & & & MED & MAD & MEL & OTROS & & \\
\hline $\begin{array}{l}\text { Smilax havanensis } \\
\text { Jacq. }\end{array}$ & $\begin{array}{r}\text { Bejuco } \\
\text { Name }\end{array}$ & & X & & $\mathrm{X}$ & $X$ & 8 & 3 \\
\hline \multicolumn{9}{|l|}{ Solanaceae } \\
\hline $\begin{array}{l}\text { Capsicum frutescens } \\
\text { L. }\end{array}$ & $\begin{array}{l}\text { Ají } \\
\text { Guaguao }\end{array}$ & & X & & & $X$ & 11 & 2 \\
\hline $\begin{array}{l}\text { Solanum erianthum } \\
\text { D. Don }\end{array}$ & $\begin{array}{l}\text { Pendejera } \\
\text { Macho }\end{array}$ & & & & & & 8 & 3 \\
\hline $\begin{array}{l}\text { Solanum havanense } \\
\text { Jacq. }\end{array}$ & Lila & & & & & $\mathrm{X}$ & 8 & 3 \\
\hline $\begin{array}{l}\text { Solanum torvum Sw. } \\
\text { Sterculiaceae }\end{array}$ & Pendejera & & $\mathrm{X}$ & & & & $1,4,8$ & 1,3 \\
\hline $\begin{array}{l}\text { Guazuma ulmifolia } \\
\text { Lam. }\end{array}$ & Guásima & & $\mathrm{X}$ & $\mathrm{X}$ & $X$ & $X$ & 6 & 2,4 \\
\hline $\begin{array}{l}\text { Hildergardia cubensis } \\
\text { (Urb.) Kosterm. }\end{array}$ & Guana & CC-COr & & $X$ & & $\mathrm{X}$ & 7,11 & 1,2 \\
\hline $\begin{array}{l}\text { Melochia nodiflora } \\
\text { Sw. }\end{array}$ & $\begin{array}{l}\text { Malva } \\
\text { Colorada }\end{array}$ & & & & $\mathrm{X}$ & & 8 & 3 \\
\hline $\begin{array}{l}\text { Melochia pyramidata } \\
\text { L.var. pyramidata }\end{array}$ & $\begin{array}{l}\text { Malva } \\
\text { Común }\end{array}$ & & & & & & 11 & 2 \\
\hline $\begin{array}{l}\text { Sterculia apetala } \\
\text { (Jacq.) Karst. }\end{array}$ & Anacagüita & & $X$ & $X$ & $\mathrm{X}$ & $\mathrm{X}$ & 9 & 1 \\
\hline \multicolumn{9}{|l|}{ Thelypteridaceae } \\
\hline $\begin{array}{l}\text { Thelypteris patens (Sw. } \\
\text { Small var. scabriuscula } \\
\text { (Presl.) A.R. Smith }\end{array}$ & & & & & & & 6 & 4 \\
\hline $\begin{array}{l}\text { Thelypteris tetragona } \\
\text { (Sw.) Small var. tetragona }\end{array}$ & & & & & & & 8 & 3 \\
\hline Tiliaceae & & & & & & & & \\
\hline $\begin{array}{l}\text { Corchorus } \\
\text { siliquosus L. }\end{array}$ & Malva Té & & $\mathrm{X}$ & & $\mathrm{X}$ & $\mathrm{X}$ & 6 & 2,4 \\
\hline $\begin{array}{l}\text { Triumfetta } \\
\text { semitriloba Jacq. } \\
\text { Ulmaceae }\end{array}$ & Guizazo & & $\mathrm{X}$ & & $\mathrm{X}$ & & 6,8 & $2,3,4$ \\
\hline Celtis trinervia Lam. & $\begin{array}{l}\text { Ramón } \\
\text { de Sierra }\end{array}$ & & & $X$ & $\mathrm{X}$ & $\mathrm{X}$ & 3 & 1 \\
\hline Urticaceae & & & & & & & & \\
\hline $\begin{array}{l}\text { Pilea trianthemoides } \\
\text { (Sw.) Lindl. }\end{array}$ & & & & & & $\mathrm{X}$ & 8 & 3 \\
\hline Verbenaceae & & & & & & & & \\
\hline $\begin{array}{l}\text { Lantana camara L. } \\
\text { var. camara }\end{array}$ & Filigrana & & $\mathrm{X}$ & & $\mathrm{X}$ & $\mathrm{X}$ & 8 & 3 \\
\hline $\begin{array}{l}\text { Priva lappulacea } \\
\text { (L.) Pers. }\end{array}$ & Farolito & & $\mathrm{X}$ & & & & 4 & 1 \\
\hline
\end{tabular}


Aportes al conocimiento de la riqueza florística para la gestión ambiental de la Sierra de Najasa, Camagüey, Cuba

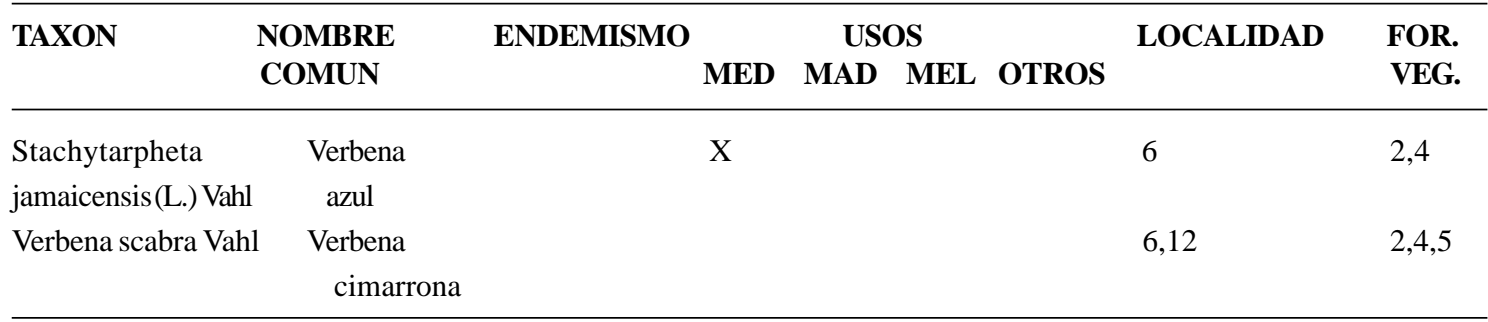

\section{REFERENCIAS BIBLIOGRÁFICAS}

Arias, R. (1994). Arboles nativos de uso múltiple utilizados por pequeños productores de Guatemala. Rev. Forestal Centroamericana 3(7):10-15.

Borhidi, A. (1976). Fundamentos de Geobotánica en Cuba. Tesis para el grado de Doctor en Ciencias Biológicas, Budapest, Hungría, 345 pp.

Borhidi, A. \& Muñiz, O. (1986). The phytogeographic survey of Cuba II. Floristic relationships and phytogeographic subdivision. Acta Botanica Hungarica 32(1-4): 3-48.

EE.UU. (1979). Tropical legumes: resources for the future. National Academy of Science, Washington DC., E.U.A., 100 pp.

Flores, J.C., Martínez, C., Olvera, M., Galván, R. \& Chávez, C. (1988). Potencial de algunas leguminosas de la Flora Yucatense como alimento humano y/o animal. Turrialba 38(2): 159-162.

Fors, A.J. (1957). Maderas Cubanas. 4ta. ed. La Habana, Cuba, 162 pp.

Fuentes, V. (1988). Las plantas medicinales de Cuba. Tesis para el grado a Doctor en Ciencias Biológicas, La Habana, Cuba.

Havard-Duclos, B. (1969). Las plantas forrajeras tropicales. I.L., La Habana, Cuba, $376 \mathrm{pp}$.

Hernández, J. \& López, M.E. (1991). Lista preliminar de plantas tintóreas que crecen en Cuba. Rev. Jardín Bot. Nac. 11(2):

Ordext, G.S. (1978). Flora apícola de la América tropical.Ed. Científico-Técnica,
La Habana, Cuba, 309 pp.

Pérez, E., Enríquez, N. \& Oviedo, R. (1994).

Características florísticas y fisionómicas de la vegetación boscosa de las sierras Najasa, Guaicanámar y Cerros Cachimbos, municipio Najasa, Camagüey, Cuba. Acta Botánica Cubana 95:1-24.

Roig, J.T. (1974). Plantas medicinales, aromáticas o venenosas de Cuba. Ed. Ciencia y Técnica, I. L., La Habana, Cuba, 949 pp.

Sobrevila, C. \& Bath, P. (1992). Evaluación ecológica rápida. Ed. Preliminar, Programa de Ciencias para América Latina, U.S.A. 